\title{
Quelle est la prochaine étape après le lancement du plan stratégique de la SCPH?
}

\author{
par Lauza Saulnier
}

$\mathrm{L}$ a Société canadienne des pharmaciens d'hôpitaux (SCPH) a présenté son nouveau plan stratégique au cours des dernières Séances éducatives d'été tenues à Ottawa en août 2015. Le plan stratégique 2015-2018, disponible au www.cshp.ca/aboutUs/ strategicPlan_e.asp, s'appuie sur la vision et les objectifs de la SCPH ainsi que sur les points à améliorer en priorité.

Le plan expose l'orientation future de l'organisation déterminée par quatre champs stratégiques clés : la pratique des pharmaciens, la mobilisation des membres et des bénévoles, la gouvernance et l'efficacité organisationnelle. Seize buts stratégiques harmonisés à ces champs stratégiques ont été définis afin de répondre aux questions suivantes :

- Quelles améliorations la SCPH s’engage-t-elle à apporter en ce qui touche aux avantages pour les membres?

- Comment la SCPH financera-t-elle ses programmes et services?

- Quels processus internes la SCPH devrait-elle améliorer si elle souhaite répondre aux attentes des membres tout en respectant ses obligations financières?

- Comment la SCPH s'assurera-t-elle que son personnel et ses bénévoles, sa culture ainsi que la technologie à sa disposition lui permettront d'atteindre ses buts stratégiques?

Transformer la vision et les priorités stratégiques d'une organisation en actions qui conduiront au résultat souhaité peut souvent être plus ardu qu'on le croit. Robert Kaplan et David Norton, créateurs du concept de tableau de bord équilibré, ont présenté un système de gestion global qui peut être employé pour exécuter efficacement un plan stratégique (Execution Premium: Linking Strategy to Operations for Competitive Advantage, Harvard Business Press, 2008). Des exemples solides de mise en pratique peuvent aussi être tirés du travail d'autres organisations nationales. Ainsi l'Association des pharmaciens du Canada a mené le Plan directeur pour la pharmacie, un projet collaboratif multilatéral dont l'objectif était d'élaborer et de concrétiser une vision pour l'avenir de la pharmacie au Canada (consultez le site Web http://plandirecteurpourlapharmacie.ca/accueil). L'Institut canadien pour la sécurité des patients a adopté le plan d'affaires «Sécurité des patients : progresser avec les quatre " pour la période 20132018, afin d'uniformiser la sécurité des patients au Canada (consultez la page http://www.patientsafetyinstitute.ca/fr/about/ patientsafetyforwardwith4/pages/default.aspx).

Pour qu'une organisation ait du succès, il est impératif que tous ses paliers épousent sa vision collective de même que son orientation stratégique et qu'ils les comprennent. Les leaders de l'ensemble de la SCPH travailleront alors à transmettre le plan stratégique aux sections provinciales, aux conseils affiliés et aux comités pour qu'ils l'adoptent et ainsi faire en sorte que leurs objectifs et leurs actions soient intégrés dans les priorités stratégiques de la Société.

Comme pour bon nombre d'organisations, les membres représentent le meilleur atout de la SCPH. La mobilisation de tous au sein de la Société dans ce processus conduira à la mise en œuvre réelle du plan stratégique, ce qui, en s'appuyant sur les forces des membres, contribuera à l'optimisation des activités locales, à la réalisation des objectifs organisationnels et à une réussite stratégique complète.

Alors que des pratiques stratégiques de gestion sont adoptées dans l'ensemble de l'association, je suis emballée à l'idée de coopérer avec chacun des membres, bénévoles et employées afin d'améliorer et de maintenir les services grandement appréciés qu'offre depuis longtemps la SCPH et de concrétiser notre vision commune.

[Traduction par l'éditeur]

Lauza Saulnier, B. Sc. (Pharm.), ACPR, est présidente désignée et agente de liaison pour la vision de la Société canadienne des pharmaciens d'hôpitaux. 